\title{
Computational Analysis of Multi-Rotor Flows
}

\author{
Seokkwan Yoon ${ }^{1}$, Henry C. Lee ${ }^{2}$, and Thomas H. Pulliam ${ }^{3}$ \\ NASA Ames Research Center, Moffett Field, California 94035
}

\begin{abstract}
Interactional aerodynamics of multi-rotor flows has been studied for a quadcopter representing a generic quad tilt-rotor aircraft in hover. The objective of the present study is to investigate the effects of the separation distances between rotors, and also fuselage and wings on the performance and efficiency of multirotor systems. Three-dimensional unsteady Navier-Stokes equations are solved using a spatially $5^{\text {th }}$ order accurate scheme, dual-time stepping, and the Detached Eddy Simulation turbulence model. The results show that the separation distances as well as the wings have significant effects on the vertical forces of quadroror systems in hover. Understanding interactions in multi-rotor flows would help improve the design of next generation multi-rotor drones.
\end{abstract}

\section{Introduction}

Recent interest in technologies that will decrease noise, emissions, and fuel burn has led to research in hybrid/full electric propulsion systems for future transport applications. The unique ability of vertical lift vehicles to hover has great potential in the civil market for human and cargo transportation, delivery systems, inspection and surveillance missions, and disaster relief.

Multi-rotors are frequently used for small electric unmanned rotorcraft partly due to their mechanical simplicity. The use of multiple motors provides redundancy as well as cost-efficiency. One of the most popular configurations is a quadrotor. Although the first quadrotor was built more than a hundred years ago, the concept has rarely been used until recently because of its inherent stability and control problems. However, recent advances in inexpensive electronic flight control systems have opened the floodgates for small drones using multi-rotors.

Small multi-rotor drones have often been designed using a hundred year old approach that is "sketch, build, fly, and iterate." In that approach, there is no systematic way to explore trade-offs or determine logical next steps. ${ }^{1}$ It is neither possible to account for multiple real-world constraints up front in design nor possible to know what performance is possible with a given design. Since unmanned vehicles are sized and optimized for particular missions, a modern low-fidelity conceptual design and sizing tool that has been used for the design of large helicopters can be used for design of small multi-rotor craft.

However, unlike most helicopters with single main rotor, the interactions between multiple rotors and a fuselage emerge as an important factor to consider in design because an increase in performance of a multi-rotor system is not simply proportional to the number of rotors. High-fidelity computational fluid dynamics (CFD) tools are required to investigate the physics of multi-rotor interactions that are not well understood at this time. High-fidelity CFD can also provide a database that may be used to calibrate a low-fidelity design tool.

Multi-rotor configurations suffer from low aerodynamic efficiencies both in hover and in cruise. ${ }^{2}$ Also, current experience with these vehicles is limited to small aircraft. It is unclear how scalable such vehicles will be, even to the modest size required for small package delivery applications. While a separate study on small drones is in progress, present work covers large-scale multi-rotors for heavy-lifting missions. Considering the recently proposed FAA drone rules that require flight only in visual line-of-sight, a large multi-rotor might be used as an airborne

\footnotetext{
${ }^{1}$ NASA Advanced Supercomputing Division

${ }^{2}$ Science and Technology Corporation

${ }^{3}$ NASA Advanced Supercomputing Division, AIAA Associate Fellow
} 
carrier for launch and recovery of unmanned aircraft systems with a human operator onboard. Compared to single rotor systems, multi-rotors offer an advantage in lifting capacity because the size of a rotor is limited by the tip speed and structural mechanics. For applications to civil operations, their aerodynamic performance and noise levels need to be assessed. Noise is one of the largest limiting factors to rotorcraft operations in urban areas. The objective of the present work is to study the effects of separation distances between rotors, and also fuselage and wings on the performance and efficiency of multi-rotor systems. The data for near-body pressure fluctuations can be used for acoustic analyses later.

\section{Numerical Approach}

Accurate prediction of rotorcraft performance in hover continues to be challenging for CFD. Flows are inherently complex because a rotor blade can encounter its own tip vortex and the tip vortices of other blades. The distance from the rotor to the far-field computational boundary has a significant effect on the figure of merit (FM) prediction. The asymptotic FM is reached when the far-field boundary is located about fifteen to twenty rotor radii away from the rotor. ${ }^{3}$ Also, it often requires twenty to thirty revolutions for a running mean of the FM to reach a quasi steady state even in free air. OVERFLOW has been used for computational simulations of multi-rotor flows.

OVERFLOW solves the Reynolds-Averaged Navier-Stokes (RANS) equations on structured overset grids. The current time-accurate approach consists of an inertial coordinate system where near-body (NB) curvilinear O-grids (rotor blades and hub) rotate through a fixed off-body (OB) Cartesian grid system. The present study employs the medium-sized XV-15 isolated rotor grid system used in Ref. 3 and Ref. 4. A uniformly spaced OB Cartesian grid surrounds the rotor blades and hub and resolves the rotor wake region of interest. Coarser "brick-grids" efficiently expand the grid system to the far field, where each successive brick grid is twice as coarse as its previous neighbor. The XV-15 isolated rotor consists of three highly twisted rotor blades and a simplified hub. Each rotor blade consists of three O-grids, one for the main rotor blade and two "cap-grids" for the inboard and outboard tips. The grid spacing normal to solid surfaces is chosen to maintain $\mathrm{y}^{+}<1$. The resolved wake region has a uniform grid spacing of $10 \% \mathrm{c}_{\text {tip }}$ (rotor blade tip chord length). Care is taken to make sure the NB grid spacing is similar to the OB grid spacing where the grids overlap.


Figure 1. a) Overset grids for a quadrotor system; b) Overset grids for a quadrotor vehicle system 
A notional quadrotor system was constructed by incorporating multiple sets of the above mentioned XV-15 rotor grid system. The XV-15 blade rotors are translated into their respective locations. The clockwise rotor system was generated by mirroring the XV-15 isolated (counter-clockwise rotating) rotor systems. The quadrotor system in Fig. 1a results in a total of 122 grids, including $48 \mathrm{NB}$ grids, and consists of approximately 195 million grid points. The collective for all the rotors was set to 10 degrees, and the precone angle was set to 1.5 degrees.

The quadrotor vehicle system incorporates two wings and a fuselage body to represent a generic quad tilt-rotor aircraft. The wings are NACA 0012 blades, and are comprised of two O-grids for the wing, and two rounded tip caps. The fuselage currently is based on an arbitrary shape designed for fixed wing research. The fuselage consists of four grids covering the nose, port, and starboard portion of the fuselage, and a fuselage end cap. The resulting grid system in Fig. 1b has 199 grids, with 60 NB grids, adding to approximately 207 million grid points.

The Navier-Stokes equations are solved using finite differences with a variety of numerical algorithms and turbulence models. ${ }^{5}$ Up to $6^{\text {th }}$-order spatial accuracy for inviscid fluxes, up to $7^{\text {th }}$-order artificial dissipation, and $2^{\text {nd }}$ order time accuracy are available. ${ }^{6}$ In this study, the Pulliam-Chaussee ${ }^{7}$ diagonal central difference algorithm is used with the $5^{\text {th }}$-order spatial differencing option. Dual time-stepping is used to advance the simulation in time with $2^{\text {nd }}$ order time accuracy. The physical time step corresponds to 0.25 degrees rotor rotation, together with 30 dual-time sub-iterations. This typically provides for a 2.5 orders of magnitude drop in sub-iteration residual. This numerical approach and time step was previously validated for similar isolated rotor flows. ${ }^{4}$ In order to reduce the computational time required for a converged solution, the first 1,440 steps employ a time step of 2.5 deg, i.e., 10 rotor revolutions. The time step is then reduced to $0.25 \mathrm{deg}$, when 1,440 steps now correspond to one rotor revolution.

Rotorcraft simulations using the Detached Eddy Simulation ${ }^{8}$ (DES) model have been successfully performed to capture the FM within experimental error. An alternative approach is to turn off the production terms in the offbody grid wake region. The Laminar Off-Body (LOB) model $^{9}$ assumes that the turbulent eddy viscosity that is generated elsewhere, e.g., the rotor blades, can still convect, but not be produced, dissipate, or diffuse. Both DES and LOB models produce accurate solutions and their FM results are comparable.,

The RANS equations require a closure by modeling the Reynolds stress. The one-equation Spalart-Allmaras ${ }^{10}$ (SA) RANS model is one of the models commonly used to compute the turbulent eddy viscosity (TEV) using the Boussinesq approximation to relate the Reynolds stresses to a kinematic turbulent eddy viscosity and the mean strain-rate tensor. The turbulent eddy viscosity is given by the expression

$$
v_{t}=\tilde{v} f_{v 1}
$$

The turbulence transport equation for the turbulence variable $\tilde{v}$, is given by

$$
\frac{D \tilde{v}}{D t}=P(\tilde{v})-D_{d i s s}(\tilde{v})+D_{d i f f}(\tilde{v})
$$

where $D / D t$ is the material time derivative. The right-hand side consists of turbulence production, dissipation and diffusion source terms. The production is given by

$$
P(\tilde{v})=C_{b 1} \tilde{v}\left(\Omega+\frac{\tilde{v}}{\kappa^{2} d^{2}} f_{v 2}\right)
$$

the dissipation by

$$
D_{\text {diss }}(\tilde{\boldsymbol{v}})=C_{w 1} f_{w}\left(\frac{\tilde{\boldsymbol{v}}}{d}\right)^{2}
$$

and the diffusion by

$$
D_{\text {diff }}(\tilde{\boldsymbol{v}})=\frac{1}{\sigma}\left[\nabla \bullet((v+\tilde{v}) \nabla \tilde{\boldsymbol{v}})+C_{b 2}(\nabla \tilde{\boldsymbol{v}})^{2}\right]
$$

The constants $\mathrm{C}_{b 1}, \mathrm{C}_{b 2}, \mathrm{C}_{w 1}, \kappa$, and functions $f_{v 1}, f_{v 2}, f_{w}$, are described by Spalart and Allmaras. ${ }^{8}$ The damping function, $f_{v 1}$, reduces $v_{t}$ near a solid wall. Note that the OVERFLOW code uses the magnitude of vorticity, $\Omega$, in place of the strain-rate, since these two are approximately equal in a boundary layer and the vorticity is readily 
available in the OVERFLOW code. The turbulence length scale, $d$, is defined as the distance from a field point to the nearest wall. The accuracy of the SA-RANS model depends strongly on the source terms in Eq. (2), which were primarily developed for attached boundary-layer flows along flat plates, wings, fuselages, etc.

The turbulence length scale, $d$, plays a key role in accurately determining the rotor FM. A problem occurs deep within the rotor wake, where $d$ may be several rotor radii in length. In this case, $d$ no longer represents an estimate of the largest turbulent eddy in the local flow, but rather, a very large geometric parameter. Note that when $d$ is very large the turbulence dissipation, Eq. (4), becomes very small. On the other hand, the strong tip vortices in the lower wake can generate significant turbulence production from Eq. (3). Over time, this imbalance in turbulence production and dissipation in the lower wake can result in excessively large eddy viscosities. These large viscosities can migrate up the vortex wake after several rotor revolutions and, under blade-vortex interaction conditions, infiltrate the blade boundary layers. When this happens, the rotor blade drag and torque increase significantly and artificially, resulting in large FM errors and an under-prediction of rotor efficiency.

The DES model is a RANS/LES hybrid approach that mitigates the problem of artificially large eddy viscosity.

The turbulence length scale used in Eqns. 1-5 is modified by replacing $d$ by $\bar{d}$ as given by Eq. (6), namely the minimum of the distance from the wall, $d$, and the local grid spacing, $\Delta=\max (\Delta \mathrm{x}, \Delta \mathrm{y}, \Delta \mathrm{z})$.

$$
\bar{d}=\min \left(d, C_{D E S} \Delta\right)
$$

The fixed coefficient is given by $C_{D E S}=0.65$. This simple but crucial change can be viewed in two different ways. From a numerical perspective the length scale in Eq. (6) has been significantly reduced which allows the turbulence dissipation in Eq. (4) to remain active in the vortex wake below the rotor plane. This prevents the turbulent eddy viscosity from growing to unrealistic values. The torque therefore remains unaffected, compared to the process described above, and the FM is accurately predicted. A physical interpretation views the modified length scale as an implicit filter, where the largest turbulent eddies are now grid-resolved on the order of size $\Delta$. All smaller eddies are modeled by a reduced turbulent eddy viscosity. This DES approach provides a rational way to reduce the length scale, and hence the turbulent eddy viscosity, based on a physical model.

The DES approach assumes that the wall-parallel grid spacing, $\Delta$, exceeds the thickness of the boundary layer so that the RANS model remains active near solid surfaces. If $\Delta<\delta$, the boundary layer thickness, then the DES Reynolds stresses can become under-resolved within the boundary layer, and this may lead to non-physical results, including grid-induced separation. Using delayed detached eddy simulation ${ }^{10}$ (DDES), the RANS mode is prolonged and is fully active within the boundary layer. The wall-parallel grid spacing used in this study does not violate the hybrid-LES validity condition. Thus DES and DDES should give similar results.

\section{Results}

The XV-15 rotors have been used for computational simulations of quadrotor systems in this study. The diameter of the XV-15 rotor is $25 \mathrm{ft}$. The Mach number at the rotor tip is 0.6902 , the Reynolds number 4.925 million based on the chord length of the rotor blade, and temperature $59 \mathrm{deg} \mathrm{F}$. The results have been obtained using the DES model for both NB and OB grids.

Figure 2 shows the Q-criterion colored by vorticity magnitude for a quadrotor flow. Here, the separation distance between the rotor tips is 30 percent of the rotor diameter $(0.3 \mathrm{D})$. The actual distance between the twin rotors in the XV-15 tilt-rotor aircraft is $0.2867 \mathrm{D}$. While the upper right and lower left rotors rotate counterclockwise, the upper left and lower right rotors rotate clockwise. This rotation direction is typical of quadcopters. Note the ingestion of vortices from above the rotors into the wakes. This phenomenon has not been observed in isolated rotors. An overhead view in Fig. 3 shows significant turbulent flow interactions between rotors. Upward flows between the rotors may be pulling the wakes toward the center. 


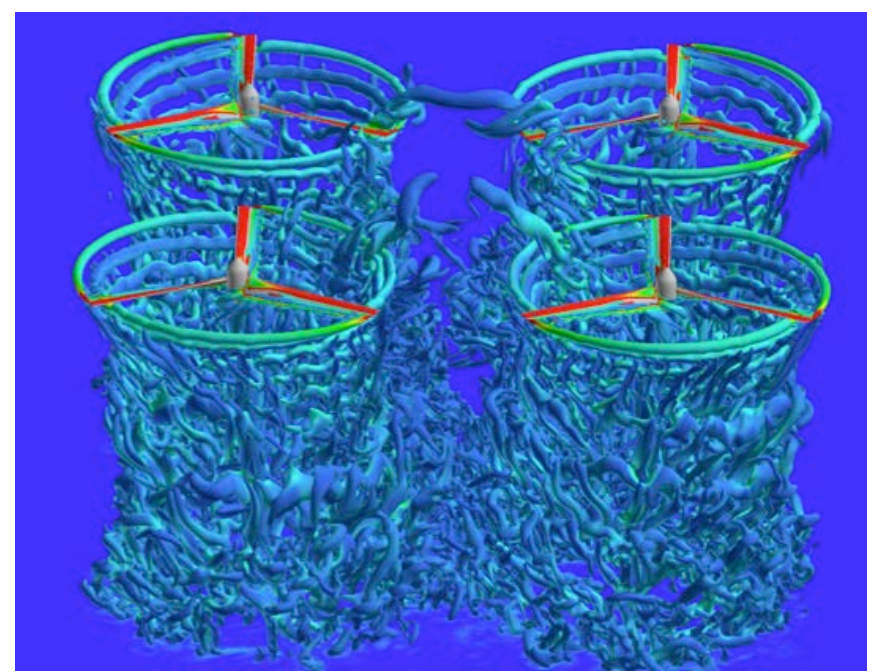

Figure 2. Q-Criterion for a quadrotor in hover. Rotor separation distance $=0.3 \mathrm{D}, \mathrm{M}_{\mathrm{tip}}=0.69, \mathrm{Re}_{\mathrm{Ctip}}=4.9$ million, $\theta=10^{\circ}$.

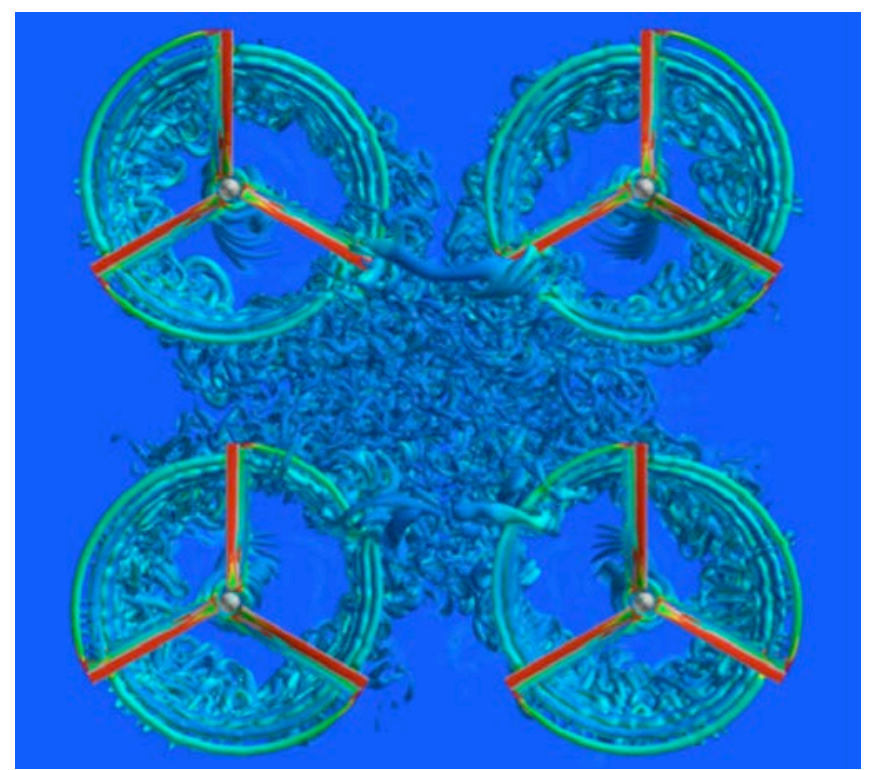

Figure 3. Q-Criterion for a quadrotor in hover (overhead view). Rotor separation distance $=0.3 \mathrm{D}, \mathrm{M}_{\mathrm{tip}}=0.69$, $\operatorname{Re}_{\text {Ctip }}=4.9$ million, $\theta=10^{\circ}$.

Figure 4 shows pressure and velocity vectors on a coordinate $(\mathrm{y}=0)$ plane between front and back rotor sets in addition to the Q-criterion. Note propagation of complex pressure waves (plot range: 0.713-0.715). They might have resulted from the interactions between the rotor blades and the ingested vortices. The estimated frequency for this case is approximately $300 \mathrm{~Hz}$. Also, the velocity vectors suggest counter-rotating vortical flows above the rotors. Figure 5 shows the results for the separation distance $0.5 \mathrm{D}$. Although pressure waves seem to be weaker than the $0.3 \mathrm{D}$ case, there still is ingestion of vortices to the wakes. 


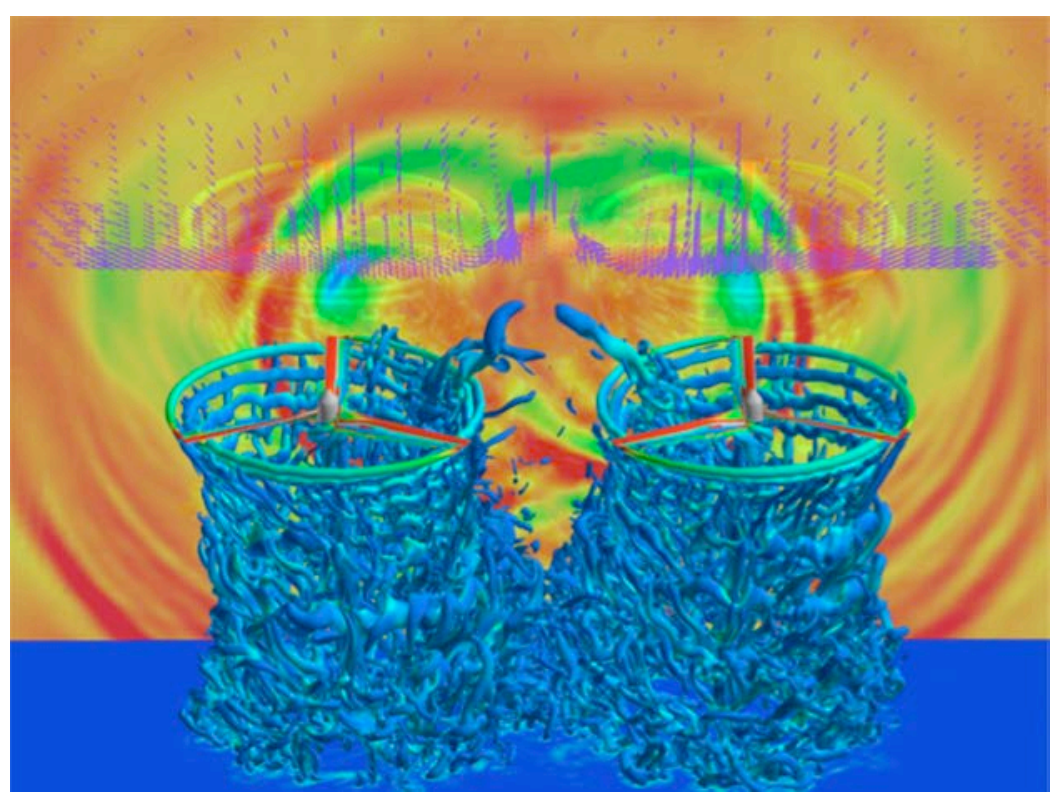

Figure 4. Q-Criterion, pressure, and velocity vectors for a quadrotor in hover. Rotor separation distance $=$ $0.3 \mathrm{D}, \mathrm{M}_{\text {tip }}=0.69, \mathrm{Re}_{\mathrm{Ctip}}=4.9$ million, $\theta=10^{\circ}$.

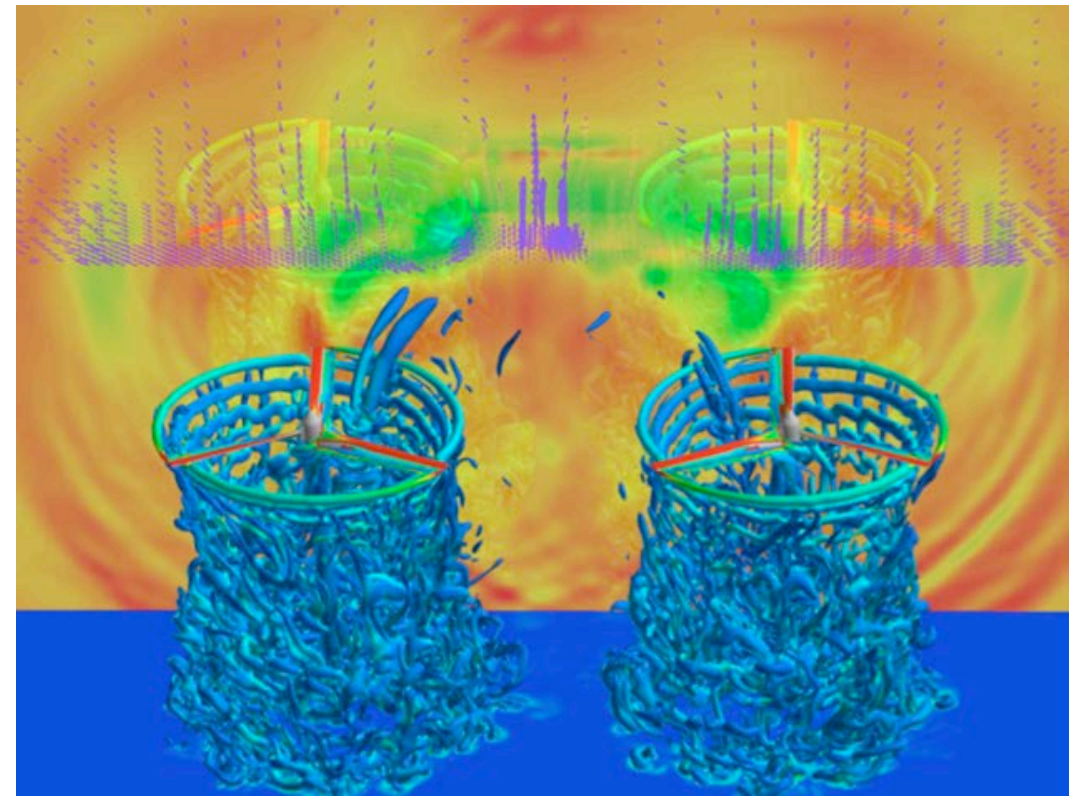

Figure 5. Q-Criterion, pressure, and velocity vectors for a quadrotor in hover. Rotor separation distance $=0.5 \mathrm{D}$, $\mathrm{M}_{\text {tip }}=0.69, \mathrm{Re}_{\text {Ctip }}=4.9$ million, $\theta=10^{\circ}$.

Figure 6 shows the results for the separation distance 1D. At this distance, the ingested vortices and highfrequency pressure waves seem to disappear. Low-frequency waves are seen instead. The estimated frequency is approximately $50 \mathrm{~Hz}$. Further apart, at a separation distance of 2D, Fig. 7 shows weakened low-frequency waves and the interaction between the rotors appear to be small. 


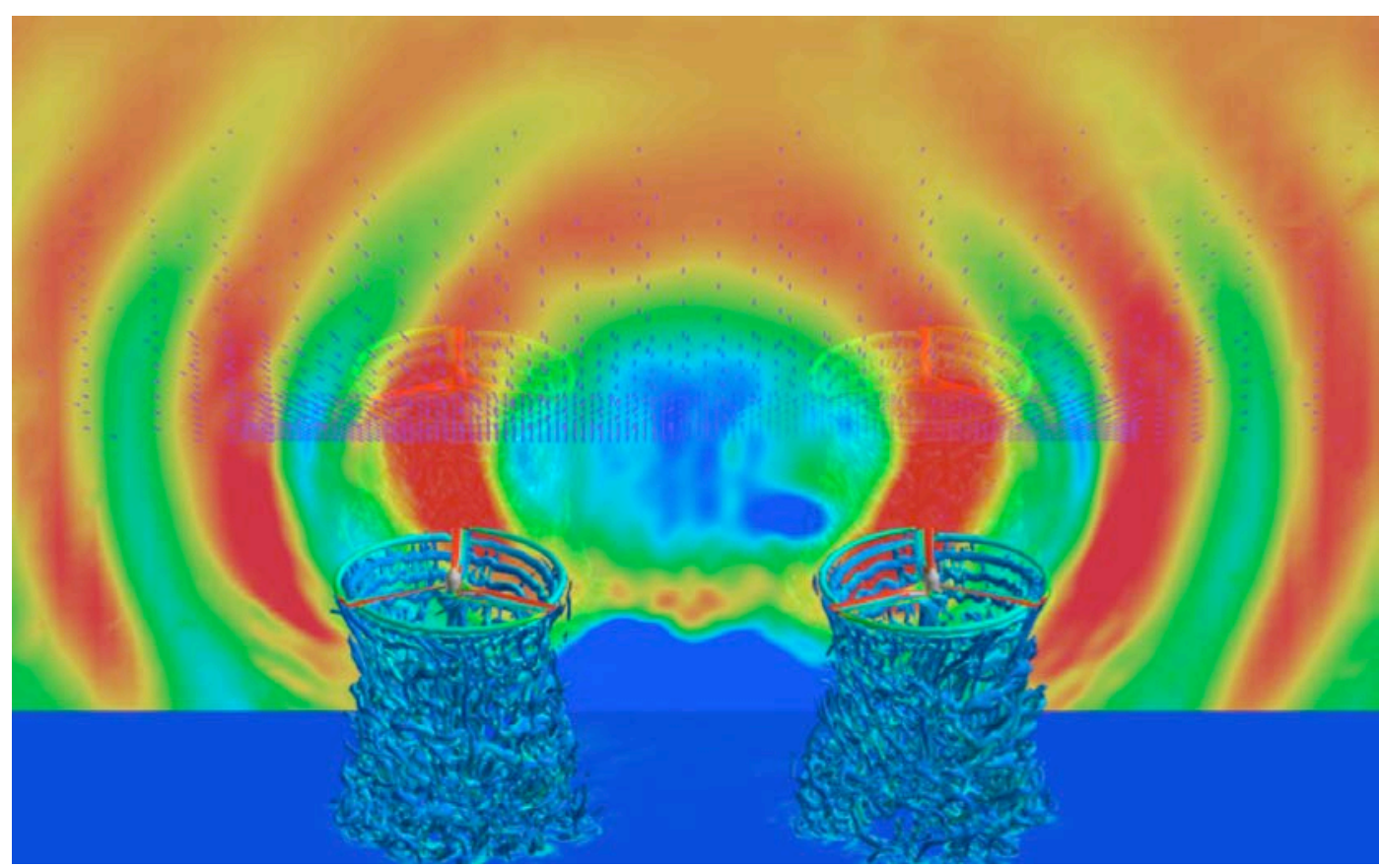

Figure 6. Q-Criterion, pressure, and velocity vectors for a quadrotor in hover. Rotor separation distance $=1.0 \mathrm{D}$, $\mathrm{M}_{\text {tip }}=0.69, \mathrm{Re}_{\text {Ctip }}=4.9$ million, $\theta=10^{\circ}$.



Figure 7. Q-Criterion, pressure, and velocity vectors for a quadrotor in hover. Rotor separation distance $=2.0 \mathrm{D}$, $\mathrm{M}_{\text {tip }}=0.69, \operatorname{Re}_{\text {Ctip }}=4.9$ million, $\theta=10^{\circ}$.

Figure 8 shows the effect of the rotor separation distance on the vertical force in hover. The average vertical force of a quadrotor (the total vertical force divided by four) is normalized by that of an isolated rotor. At a 
separation distance of $2 \mathrm{D}$, the average vertical force of the quadrotor appears to be close to that of an isolated rotor. However, as the separation distance gets smaller, the vertical force of the quadrotor decreases. At the separation distance of $0.3 \mathrm{D}$, the decrease in rotor efficiency is approximately 2 percent.

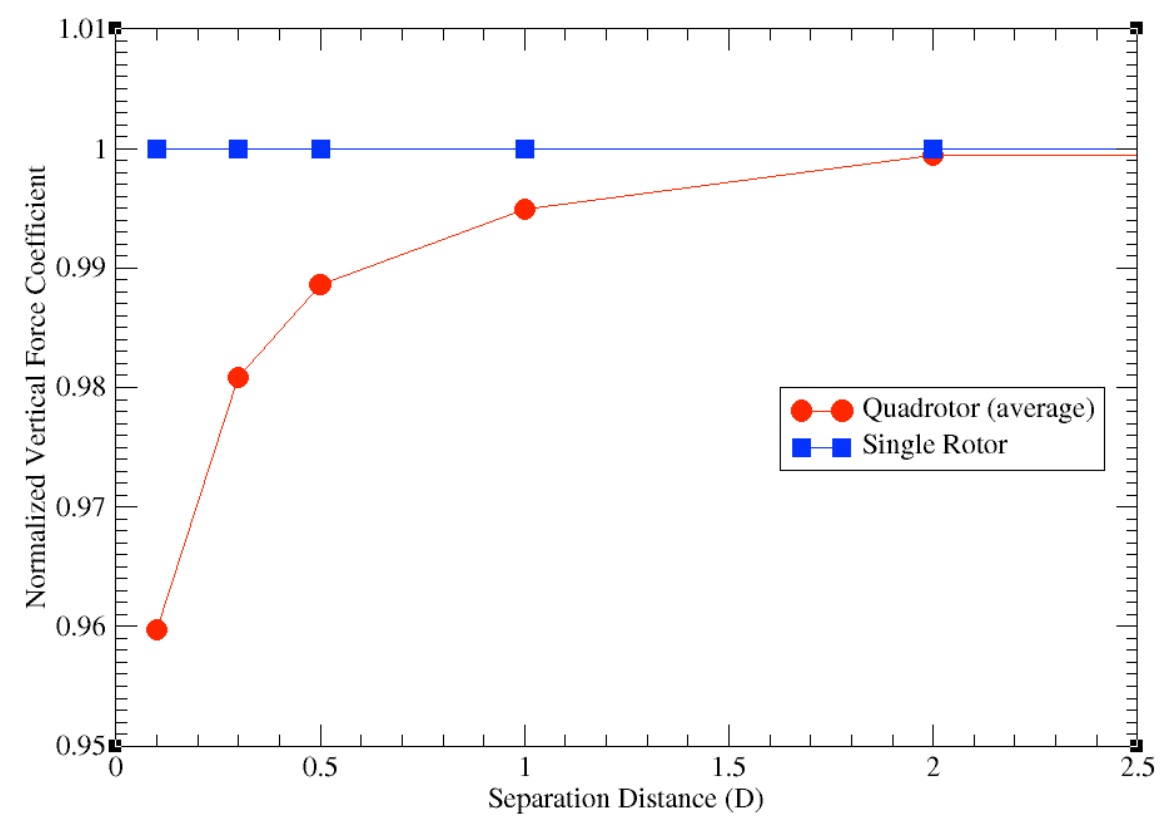

Figure 8. Effect of rotor separation distance on vertical force in hover. Average quadrotor vertical force normalized by that of an isolated rotor, $\mathrm{M}_{\text {tip }}=0.69, \mathrm{Re}_{\text {Ctip }}=4.9$ million, $\theta=10^{\circ}$.

In order to investigate multiple rotor interactions for a full rotorcraft, a fuselage and two wings have been added to a quadrotor with the separation distance $0.3 \mathrm{D}$. This represents a generic quad tilt-rotor configuration in hover. Although rotors on rear wings turn the same direction as on front wings for a quad tilt-rotor, the rotation direction typical of quadcopters is used here to quantify the effect of fuselage and wings. It should also be noted that, with realistic quad tilt-rotors, the rear wing is longer and higher than the front wing, and the rear rotors are outboard of the front rotors for higher performance and fuel economy in cruise. ${ }^{11}$

Figure 9 shows Q-criterion and surface pressure. Pressure on a horizontal plane below the vehicle is plotted as well to show the propagation of waves (note: a different color map is used for contrast). The interactions in the wakes appear to be less strong than in the case without a fuselage because the fuselage blocks the interaction of the wakes. Note the fountain flows above the fuselage just in front of both wings. The fountain flow, which was observed in a smoke visualization test (see Fig. 10), ${ }^{12}$ creates a large downward force and non-uniform distribution of noise. The rotor downwash impinges upon the top of the wing surface leading to both chordwise and spanwise flow. The chordwise flow separates at the leading and trailing edges, resulting in a separated flow below the wing. The spanwise flow moves along the upper surface inwards toward the root of the wing until it reaches the centerline. There it encounters the flow from the other side of the vehicle and deflected upwards, resulting in a fountain flow at the center of the vehicle. ${ }^{13}$ 




Figure 9. Q-Criterion and pressure for a generic quad tilt-rotor in hover. Rotor separation distance $=0.3 \mathrm{D}, \mathrm{M}_{\mathrm{tip}}=0.69$, $\operatorname{Re}_{\text {Ctip }}=4.9$ million, $\theta=10^{\circ}$.

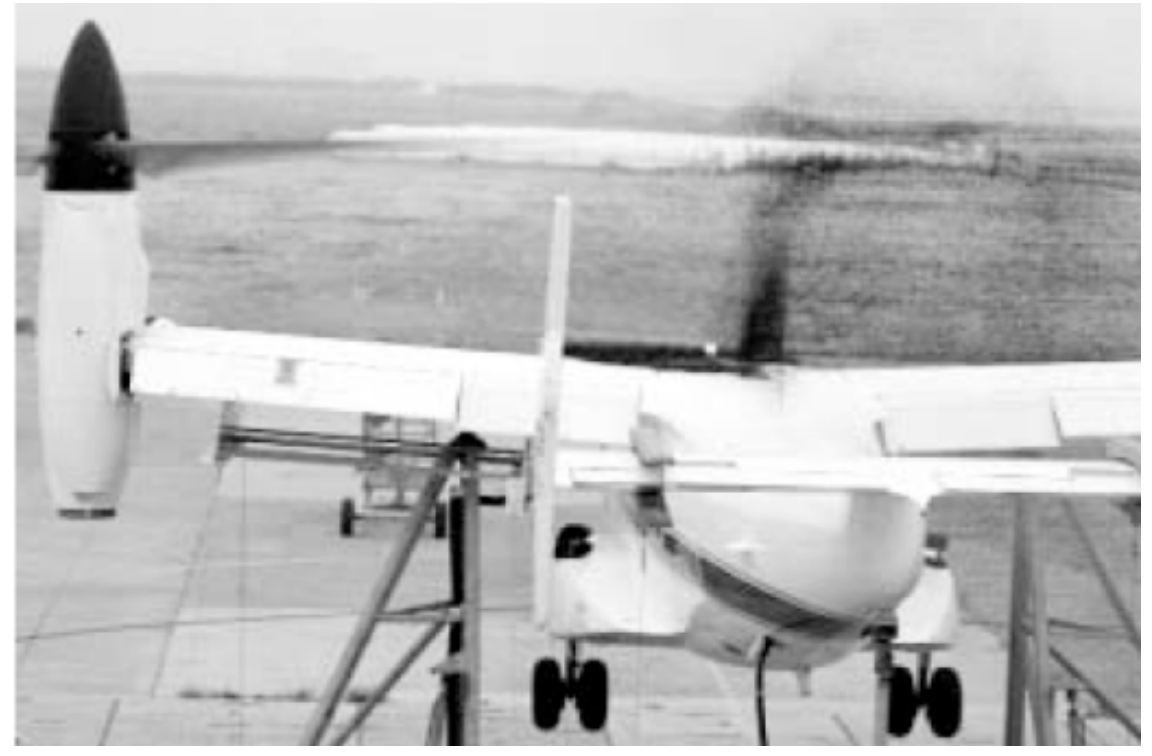

Figure 10. Smoke visualization showing fountain flow above fuselage (Ref. 11) 
A breakdown of vertical force components is shown in Fig. 11. Here, the vertical forces are normalized by $4 \mathrm{x}$ the isolated rotor force. It is interesting to note that the normalized force for rotors only is 1.029 , indicating the quadrotor in this vehicle configuration generates about 3\% higher thrust than that of four isolated rotors (or 5\% higher than that of the quadrotor without fuselage or wings). However, the wings create a large download (-0.188) and the fuselage a very small download $(-0.003)$. Thus, the total vertical force for a vehicle is 0.838 , or about $16 \%$ lower than four isolated rotors. Tilt-rotors can reduce download by lowering flaps to $80 \mathrm{deg}$ or so. Furthermore, although not included here, a flow simulation movie shows extremely unsteady fluctuations of pressure on the fuselage and wings.

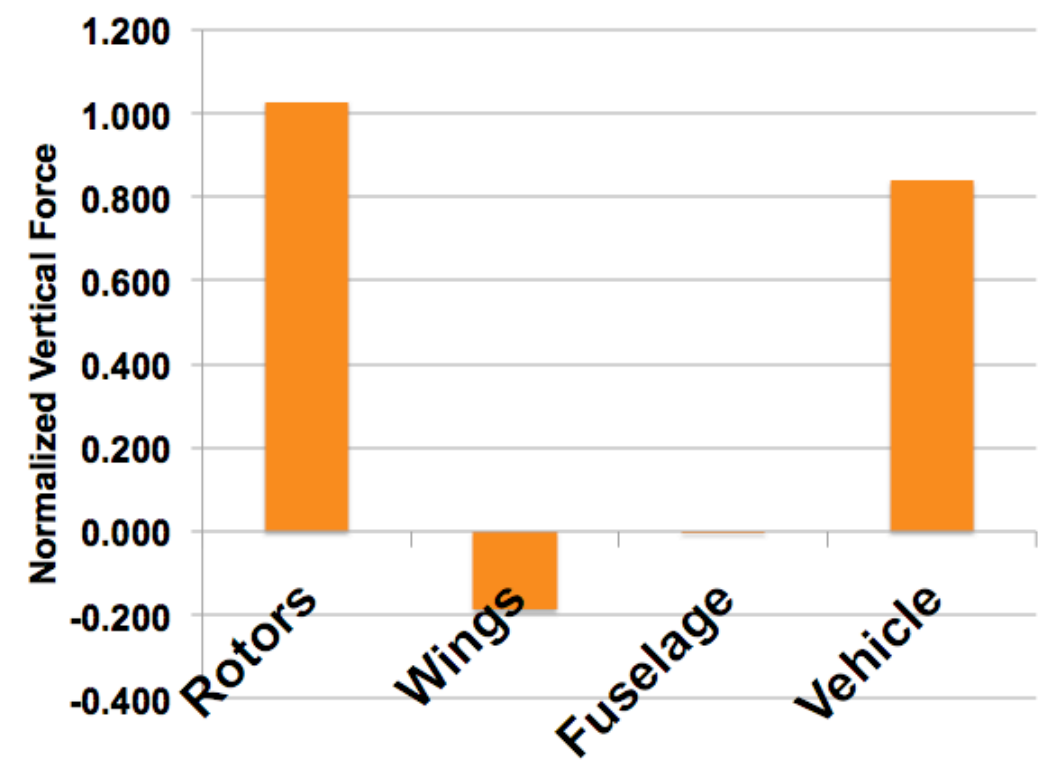

Figure 11. Breakdown of vertical force components for a quadrotor vehicle in hover; the vertical forces are normalized by $4 \mathrm{x}$ the isolated rotor force. Separation distance $=0.3 \mathrm{D}, \mathrm{M}_{\mathrm{tip}}=0.69, \operatorname{Re}_{\mathrm{Ctip}}=4.9$ million, $\theta=10^{\circ}$. Forces: Rotors (1.029), Wings (-0.188), Fuselage (-0.003), and Total Vehicle System (0.838).

\section{Conclusions}

A computational study of the interactional aerodynamics of multi-rotor flows has revealed that the rotor separation distance has a significant effect on the vertical force of a quadrotor in hover. As the separation distance between rotors gets smaller, the efficiency of the quadrotor system decreases. Whereas the fuselage itself creates little force, it seems to have a positive effect on rotor efficiency by limiting the interaction of rotor wakes. Also, the wings appear to lower the total vertical force of the quadrotor vehicle by generating a large download force.

\section{Acknowledgments}

This work was supported by the RVLT and DELIVER projects. The first author thanks Ethan Romander, Nagi N. Mansour and Wayne Johnson for helpful discussions. 


\section{References}

${ }^{1}$ Theodore, C., "Vertical Lift Hybrid Autonomy," The VLHA Meeting, NASA Ames Research Center, May 2015.

${ }^{2}$ Young, L. A., "Conceptual Design Aspects of Three General Sub-Classes of Multi-Rotor Configurations: Distributed, Modular, and Heterogeneous," The $6^{\text {th }}$ AHS Specialists Meeting on Unmanned Rotorcraft Systems, Scottsdale, AZ, Jan. 2015.

${ }^{3}$ Yoon, S., Pulliam, T. H., and Chaderjian, N. M., "Simulations of XV-15 Rotor Flows in Hover Using OVERFLOW," The $5^{\text {th }}$ Decennial AHS Aeromechanics Specialists' Conference, San Francisco, CA, Jan. 2014.

${ }^{4}$ Yoon, S., Chaderjian, N. M., Pulliam, T. H., and Holst, T. L., "Effect of Turbulence Modeling on Hovering Rotor Flows," AIAA Paper 2015-2766, The 45 ${ }^{\text {th }}$ AIAA Fluid Dynamics Conference, Dallas, Texas, June 2015.

${ }^{5}$ Nichols, R., Tramel, R., and Buning, P., "Solver and Turbulence Model Upgrades to OVERFLOW2 for Unsteady and High-Speed Flow Applications," AIAA Paper 2006-2824, June 2006.

${ }^{6}$ Pulliam, T. H., "High Order Accurate Finite-Difference Methods: as seen in OVERFLOW," AIAA Paper 20113851, June 2011.

${ }^{7}$ Pulliam, T. H., and Chaussee, D. S., "A Diagonal Form of an Implicit Approximate-Factorization Algorithm," Journal of Computational Physics, Vol. 39, No. 2, 1981, pp. 347-363.

${ }^{8}$ Spalart, P. R., W-H. Jou, Strelets, M., and Allmaras, S. R., "Comments on the Feasibility of LES for Wings and on a Hybrid RANS/LES Approach," Advances in DNS/LES, Greyden Press, 1997, pp. 137-147.

${ }^{9}$ Potsdam, M. and Pulliam, T. H., "Turbulence Modeling Treatment for Rotorcraft Wakes," AHS Aeromechanics Specialists Meeting, Jan. 2008.

${ }^{10}$ Spalart, P. R. and Allmaras, S. R., “A One-Equation Turbulence Model for Aerodynamic Flows,” AIAA Paper 1992-0439, Jan. 1992.

${ }^{11}$ Radhakrishnan, A. and Schmitz, F. H., "An Experimental Investigation of a Quad Tilt Rotor in Low Speed Forward Flight," The $4^{\text {th }}$ AHS Decennial Specialist's Conference on Aeromechanics, San Francisco, CA, Jan. 2004.

${ }^{12}$ Maisel, M. D., Giulianetti, D. J. and Dugan, D. C., "The History of the XV-15 Tilt Rotor Research Aircraft," NASA SP-2000-4517, 2000.

${ }^{13}$ Gupta, V. and Baeder, J. D., "Quad Tilt Rotor Aerodynamics in Helicopter Mode," The $61^{\text {st }}$ AHS Annual Forum, Grapewine, TX, June 2005. 